\title{
CRISPR/Cas9-mediated LMP1 knockout inhibits Epstein-Barr virus infection and nasopharyngeal carcinoma cell growth
}

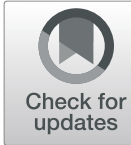

Haifeng Huo and Guohua Hu*

\begin{abstract}
Background: A strong association between Epstein-Barr virus (EBV) infection and nasopharyngeal carcinoma (NPC) has been widely recognized in recent decades. The aim of the present study was to investigate latent membrane protein 1 (LMP1) regulation of nasopharyngeal carcinoma (NPC) CNE-2 cell growth and then examine the effects of LMP1-knockout with CRISPR (Clustered Regularly Interspaced Short Palindromic Repeats)/Cas9on Epstein-Barr virus (EBV) infection and CNE-2 cell growth.
\end{abstract}

Methods: Human NPC CNE-2 cells were infected with the recombinant LMP1- and LMP2A-carrying lentivirus, and then examined for cell growth with the colony forming assay as well as for the activation of transcription of eukaryotic translation initiation factor $4 \mathrm{E}$ (elF4E) with reverse-transcriptase quantitative polymerase chain reaction (RT-qPCR) and western blot. CRISPR/Cas9-mediated knockout of LMP1 or LMP2A was performed with a single-guide RNA (sgRNA) targeting sequences within LMP1 or LMP2A. The knockout effect and the EBV proliferation were examined with RT-qPCR, western blot and cell growth assay.

Results: LMP1 overexpression promoted CNE-2 cell growth, compared to LMP2A overexpression. Loss-of-function experiments confirmed that eukaryotic translation initiation factor 4E (elF4E) upregulation mediated this effect. LMP1 knockout significantly inhibited EBV proliferation in CNE-2 cells and markedly inhibited LMP1-mediated promotion of cell growth. The knockout of either LMP1 or LMP2A blocked the elF4E activation, which is induced either by the EBV infection or by the overexpression of LMP1 or LMP2A.

Conclusion: We confirmed the LMP1-mediated promotion of NPC cell growth. Such promotion can be effectively blocked by CRISPR/Cas9-mediated LMP1 knockout. Precise LMP1 knockout might be a promising method for targeted inhibition of EBV infection and NPC cell growth.

Keywords: CRISPR/Cas9, Nasopharyngeal carcinoma, Epstein-Barr virus (EBV), LMP1, Growth

\section{Background}

A strong association between Epstein-Barr virus (EBV) infection and nasopharyngeal carcinoma (NPC) has been widely recognized in recent decades [1-4]. Classified as a group I carcinogen by the International Agency for Research on Cancer (IARC), EBV is detected in almost all poorly differentiated NPC cases [3, 4]. Oncogenic factors in NPC have been reduced to viral proteins such as latent membrane protein 1 (LMP1) $[5,6]$ and Epstein-Barr nuclear antigen 1 (EBNA1) $[4,7,8]$. The EBV-encoded small

\footnotetext{
* Correspondence: 3109595294@qq.com

Department of Otorhinolaryngology, The First Affiliated Hospital of Chongqing Medical University, Chongqing 400016, China
}

RNAs (EBERs) [9], and microRNAs are also associated with EBV-driven oncogenesis, such as miRNAs of BERTs (EBV-encoded miRNAs in PART region) [10]. Chromosomal integration of EBV genomes has been sporadically observed in NPC cells $[11,12]$. Regarding the molecular mechanisms of LMP1-driven oncogenesis in NPC, multiple signaling pathways have been found involved, such as nuclear factor $\mathrm{kB}(\mathrm{NF}-\mathrm{kB})$ [6], p38 mitogen activated protein kinase and the cJun N-terminal kinase (JNK) pathways [13-15]. The oncogenic role of LMP1 has been widely investigated, especially how it can promote epithelialmesenchymal transition (EMT) and NPC cell 
proliferation and invasion. It positively regulates TAZ expression [16], stimulates the transcription of eukaryotic translation initiation factor 4E (eIF4E) [17], upregulates high mobility group box 1 (HMGB1), facilitates EBV-LMP1targeted DNAzyme-induced DNA damage to cause cell cycle arrest [18], and inhibits the liver kinase B1 (LKB1)-AMP-activated protein kinase (AMPK) pathway [19].

CRISPR (Clustered Regularly Interspaced Short Palindromic Repeats)/Cas (CRISPR-associated) was recently verified as a precise and robust strategy for targeted genome editing [20-22]. Most commonly, Cas9 endonuclease and a single-guide RNA (sgRNA) are utilized to target a 20-bp-long DNA region that is complementary to the sgRNA [21, 23]. CRISPR/Cas9 technology enables loss-offunction genetic analysis of regulatory elements in the coding or non-coding region of a gene $[24,25]$ and robust potential for genetic modification. The CRISPR/Cas9 system has been applied to develop various antiviral strategies [26, 27], including against human herpesvirus (HHV) $[28,29]$. This strategy is more effective than other antivirus methods, particularly for viruses that integrate into human chromosomes, such as human immunodeficiency virus (HIV) and human papillomavirus (HPV) [30]. It was also demonstrated to effectively eliminate EBV genomic episomes from latent cells [31, 32].

The present study aimed to evaluate the effect of CRISPR/Cas9-mediated knockout of LMP1 or LMP2A on CNE-2 cell proliferation.

\section{Methods}

\section{Cell culture and CRISPRS-Cas9 treatment}

Human NPC CNE-2 cells were cultured in RPMI1640 medium (Thermo Fisher Scientific, Waltham, MA, USA), with $10 \%$ fetal bovine serum (FBS, Thermo Fisher Scientific, Waltham, MA, USA) and with $1 \mathrm{x}$ penicillin/streptomycin solution (Invitrogen/ Thermo Fisher Scientific). Cell incubation was performed at $37^{\circ} \mathrm{C}$ under $5 \%$ carbon dioxide $\left(\mathrm{CO}_{2}\right)$. To overexpress LMP1 or LMP2A, 85\%-confluent CNE-2 cells were infected with the recombinant LMP1- or (and) LMP2A-carrying lentivirus (Cqwestern. Chongqing, PR China), and with chloramphenicol acetyl transferase (CAT)-carrying lentivirus, cloned from pcDNA3.1/CAT, as the up control. CNE-2 cells were infected with a multiplicity of infection (MOI) of 10 and then were selected under puromycin pressure $(2 \mu \mathrm{g} / \mathrm{ml})$ for ten days. CNE-2 cells were infected with EBV (0.01 MOI), before the examination of the CRISPR/Cas9-mediated inhibition to virus proliferation, to LMP1/ LMP2A expression, or to eIF4E activation,

For CRISPR/Cas9-mediated knockout of LMP1 or LMP2A, the sgRNA targeting sequences were designed targeting potential 20-base-long sites on the EBV genome with the online gRNA design tool (Crispr.mit.edu). sgRNA sequences were listed as follows: LMP1 (targeting sequence 5' - TTG AGA GGG GCC CAC CGG GCC CG-3' [14-36 in LMP1 coding region] and 5'-CGC CTT TGA TGA CAG ACG GAG GC-3' [1007-1029 in LMP1 coding region]); LMP2A (targeting sequence 5 '-GCC GTT ACG TGT CCC GGG TGG TC-3' [14-36 in LMP2A coding region] and 5'-TGC CTC AGT GGA CTT CTC ACC GC-3' [12311253 in LMP2A coding region]). The sgRNA sequences were inserted into the sgRNA-expression vector PX459, and the gRNA-PX459 recombinant plasmids were cotransfected with gRNA-Cas9 co-expression plasmid PX459 using Lipofectamine 3000 (Invitrogen/Thermo Fisher Scientific). Positive cell clones were selected from the transfected cells at $48 \mathrm{~h}$ post transfection under the pressure of $1 \mu \mathrm{g} / \mathrm{ml}$ puromycin for two weeks, via serial passages.

\section{Reverse-transcriptase quantitative polymerase chain reaction (RT-qPCR)}

Total mRNA was extracted from CNE-2 cells with a magnetic mRNA Isolation Kit (New England Biolabs, Ipswich, MA USA) according to the manufacturer's protocol. Equivalent amount of mRNA sample was transcribed into cDNA with a GoScript ${ }^{\mathrm{Th}}$ Reverse Transcriptase (Promega, Madison, WI, USA). EBV viral DNA was purified with genomic DNA isolation kits (Qiagen, Venlo, Netherlands) under the guidance of the kit's instruction. The cDNA was examined by RT-qPCR (PrimeScript ${ }^{\mathrm{Tu}}$ RT Reagent Kit, Takara, Tokyo, Japan) to determine the relative mRNA levels of LMP1, LMP2A, and eIF4E using $\beta$-actin for gene expression normalization with LightCycler 2.0 (Roche Diagnostics, Risch-Rotkreuz, Switzerland). To quantify EBV genomic DNA in CNE cells after EBV infection, the levels of EBNA1 and OriP were quantified with RT-qPCR. The EBNA1 or OriP DNA level is presented as a relative value to each DNA level at 0 - $h$ post-EBV infection.

\section{Western blot analysis}

CNE-2 cells were lysed with Cell Lysis Buffer (Cell Signaling Technology, Danvers, MA, USA) and supplemented with protease inhibitor cocktail kit (Roche Biochemicals, Penzberg, Germany). Protein samples were separated by $10 \%$ sodium dodecyl sulfatepolyacrylamide gel electrophoresis and then transferred onto polyvinylidene fluoride membranes. The membrane was incubated with primary antibodies for LMP1 - (1:2000, Abcam, Cambridge, UK), LMP2A(1: 1000, Abcam), eIF4E-specific (1: 1000, Abcam) or $\beta$-actin (1: 3000, Sino Biological, Wayne, PA, USA). Horseradish peroxidase (HRP)-linked secondary antibodies (Jackson ImmunoResearch, West Grove, PA, USA) and enhanced chemiluminescence (Thermo Fisher Scientific) were utilized to visualize the specific binding of the HRP-linked 
second antibody to first antibody-protein complexe, under the UVP BioSpectrum 500 imaging system (UVP, Upland, CA, USA).

\section{Colony-forming assay and cell growth assay}

CNE-2 cells, with LMP1- or LMP2A-overexpression or with LMP1- and/or LMP2A-knock out, were seeded and incubated in six-well plates for three days. Colony formation was observed under an Olympus BX60 microscope (Olympus, Tokyo, Japan). Cell growth was assessed with CCK-8 assays (DOJINDO, Tokyo, Japan). Briefly, CNE-2 cells were suspended $(100 \mu \mathrm{l} /$ well $)$ in a 96-well plate and incubated in a humidified incubator (at $37^{\circ} \mathrm{C}$ with $5 \% \mathrm{CO}_{2}$ ) for 24,48 , or $72 \mathrm{~h}$. Next, $10 \mu \mathrm{l}$ CCK-8 solution was added to each well for 2-h's incubation at $37^{\circ} \mathrm{C}$, and then absorbance at $450 \mathrm{~nm}$ was measured using a microplate reader (Bio-Rad, Hercules, CA, USA).
All experiments were approved by the Ethics Committee of Chongqing Medical University (20170121).

\section{Statistical analysis}

Quantitative results are presented as mean \pm standard deviation and were analyzed with two-tailed Student's ttests. Differences were considered significant at $p<0.05$.

\section{Results}

EBV LMP1 promotes NPC CNE-2 cell growth in an elF4Edependent fashion

We overexpressed LMP1 or LMP2A in CNE-2 cells with lentiviral vectors, using CAT-carrying lentivirus as the upregulation control (abbreviated as up control). Figure 1a shows dramatically increased mRNA levels of LMP1 and LMP2A in the LMP1 up-regulation (LMP1 up) and LMP2A up-regulation (LMP2A up) groups, respectively, in comparison with the Up-control group (both $p<0.001$ ). Both LMP

\section{A}

B
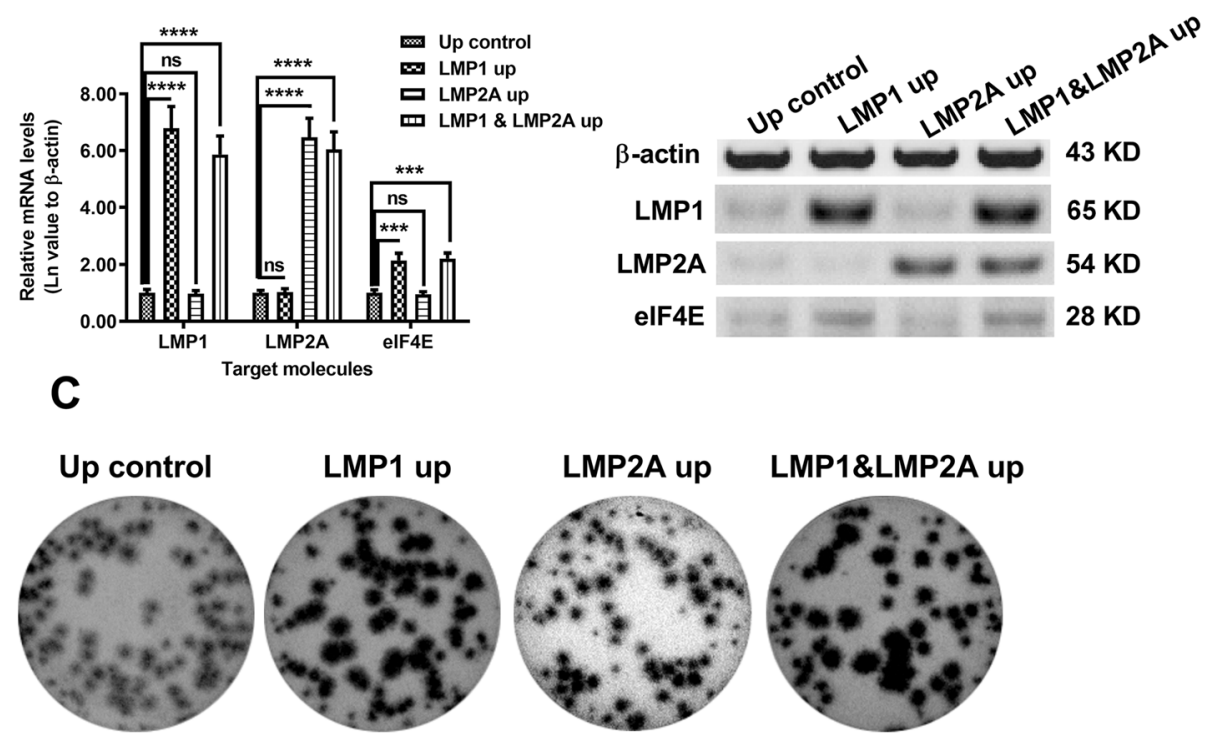

D

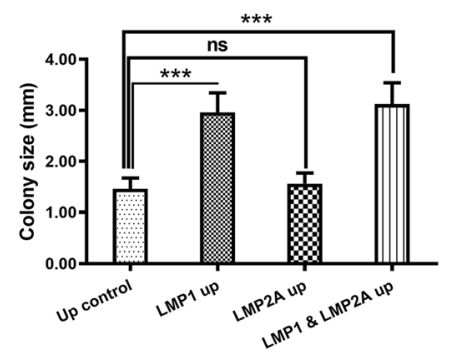

Fig. 1 LMP1 overexpression regulates CNE-2 cell growth. $\mathbf{a}$ and $\mathbf{b}$ : Real-time quantitative PCR analysis (a) and western blotting (b) of mRNA and protein levels of LMP1, LMP2A, and elF4E in the control, LMP1-overexpressing, LMP2A-overexpressing or both LMPs-overexpressing CNE-2 cells; $\mathbf{c}$ and $\mathbf{d}$ : Colony formation and colony size for CNE-2 cells after 3 days of incubation, measured via colony forming assays. Up control, control; LMP1 up, LMP1-overexpressed; LMP2A up, LMP2A-overexpressed; LMP1\&LMP2A up, both LMP1- and LMP2A overexpressed. Each result was repeated independently in triplicate. ${ }^{* * *} p<0.001 ;{ }^{* * *} p<0.0001$; ns, no significance 
proteins were upregulated in CNE-2 cells following LMP1and LMP2A-lentivirus infection (both $p<0.0001$, Fig. 1b). Also, higher eIF4E mRNA levels were observed in LMP1-overexpressing CNE-2 cells with or without LMP2A overexpression $(p<0.001$ Fig. 1a). Higher eIF4E protein level was also found in CNE-2 cells (Fig. 1b). To evaluate the LMP regulation of NPC cell growth, we performed colony-forming assays in $\mathrm{CNE}$ 2 cells followingLMP1- and LMP2A-lentivirus infection. As indicated in Fig. 1c, significantly larger colonies were formed by CNE-2 cells after LMP1lentivirus infection compared to LMP2A- as well as LMP1\&LMP2A-lenticirus infection $(p<0.001$ for the LMP1 up and LMP1\&LMP2A up groups, Fig. 1d).

Besides, growth curves confirmed that LMP1 increases cell growth $(p<0.0001$, Fig. 2a). We evaluated whether LMP1mediated promotion of CNE-2 cell growth is dependent on eIF4E. Effective knockdown of eIF4E $(p<0.001$, Fig. 2b) significantly inhibited growth promotion in CNE-2 cells $(p<0.05$, Fig. $2 \mathrm{c}$ and d). Thus, LMP1-mediated promotion of NPC cell growth was observed in CNE2 cells and was at least partly dependent on eIF4E.

\section{CRISPR/Cas9-mediated knockout of LMP1 or LMP2A inhibits EBV replication}

To explore the potential of CRISPR/Cas9-mediated knockout of EBV oncogenic proteins, LMP1- and LMP2A-targeted gRNA sites and the schematic diagram of the constructed LMP1- and LMP2A-editing systems were shown in Fig. 3a. The conservation of the target sites was checked via aligning the target sequences with other viral coding sequences. The successful insertion and effective transfection of either gRNA1 + 2 (LMP1) / gRNA1 + 2 (LMP2A) or the control plasmid were indicated by the expression of green fluorescence protein, at the 12-h post transfection (Fig. 3b). The editing effectiveness of both LMP targets is shown in Fig. $3 \mathrm{c}$ and $\mathrm{d}$ under the same transfection efficiency.

\section{A}

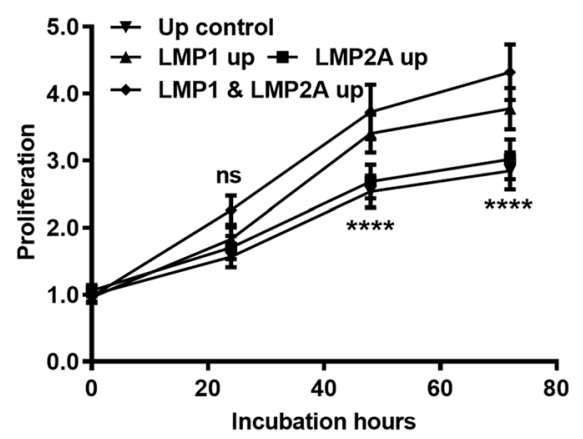

C
LMP1 up + SIRNA-elF4E

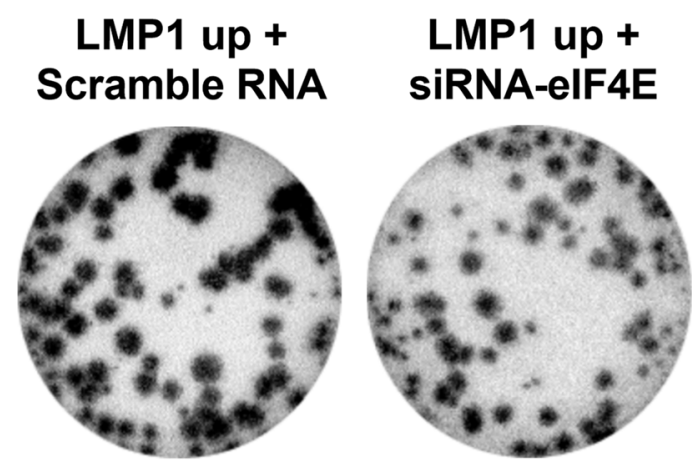

B

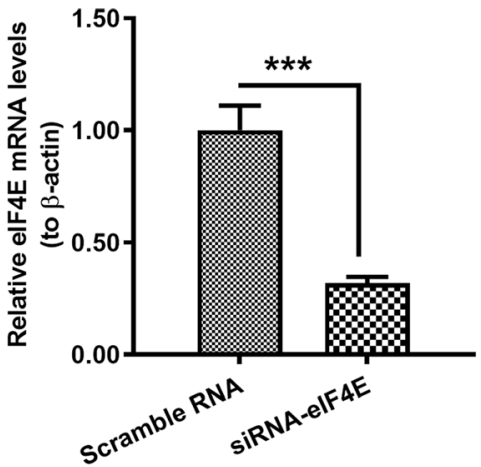

D

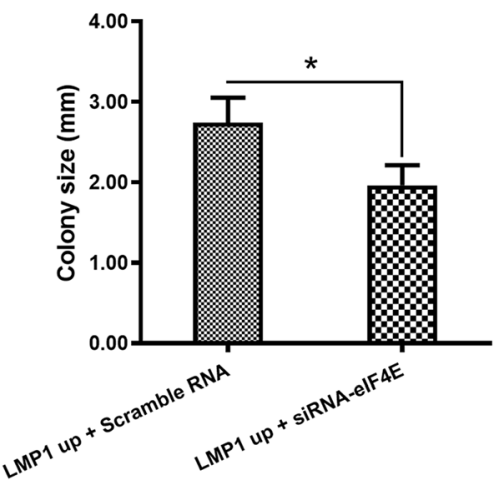

Fig. 2 Dependence of eukaryotic translation initiation factor 4E on the LMP1-mediated regulation on CNE-2 cell growth. a: Growth curves of the four groups of CNE-2 cells incubated for 24, 48, and $72 \mathrm{~h}$. $\mathbf{b}$ : Relative elF4E mRNA levels in LMP1-overexpressing CNE-2 cells after the elF4E knockdown. $\mathbf{c}$ and $\mathbf{d}$ : Colony formation and colony size for LMP1-overexpressing CNE-2 cells after the elF4E knockdown. Scramble RNA, negative control for siRNA-elF4E; siRNA-elF4E: elF4E-specific siRNA. Each result was repeated independently in triplicate. ${ }^{*} p<0.05$; ${ }^{* * *} p<0.001$; ${ }^{* * * *} p<$ $0.0001 ;$ ns, no significance 


\section{A}
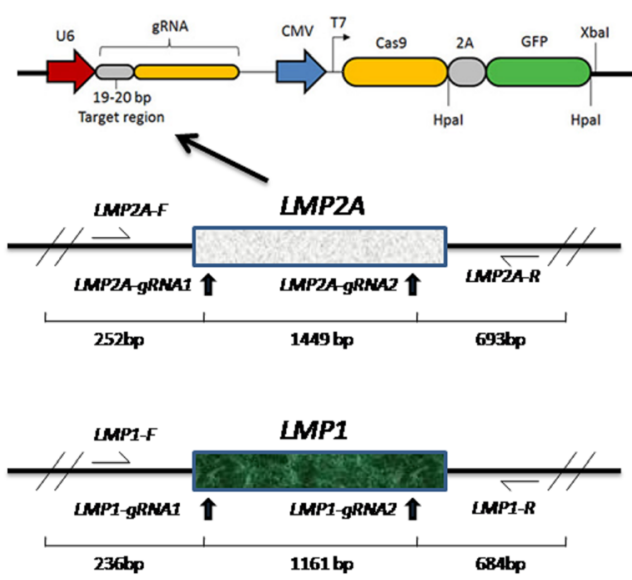

B

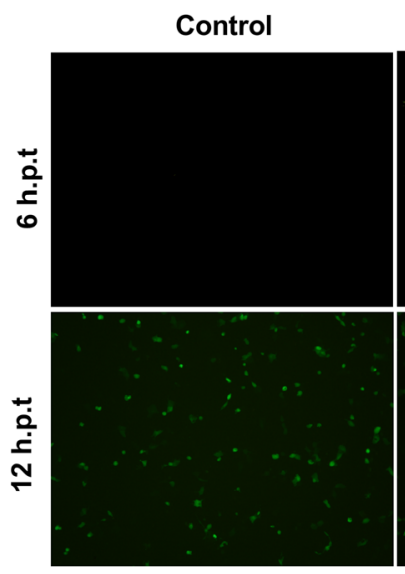

$\mathbf{E}$
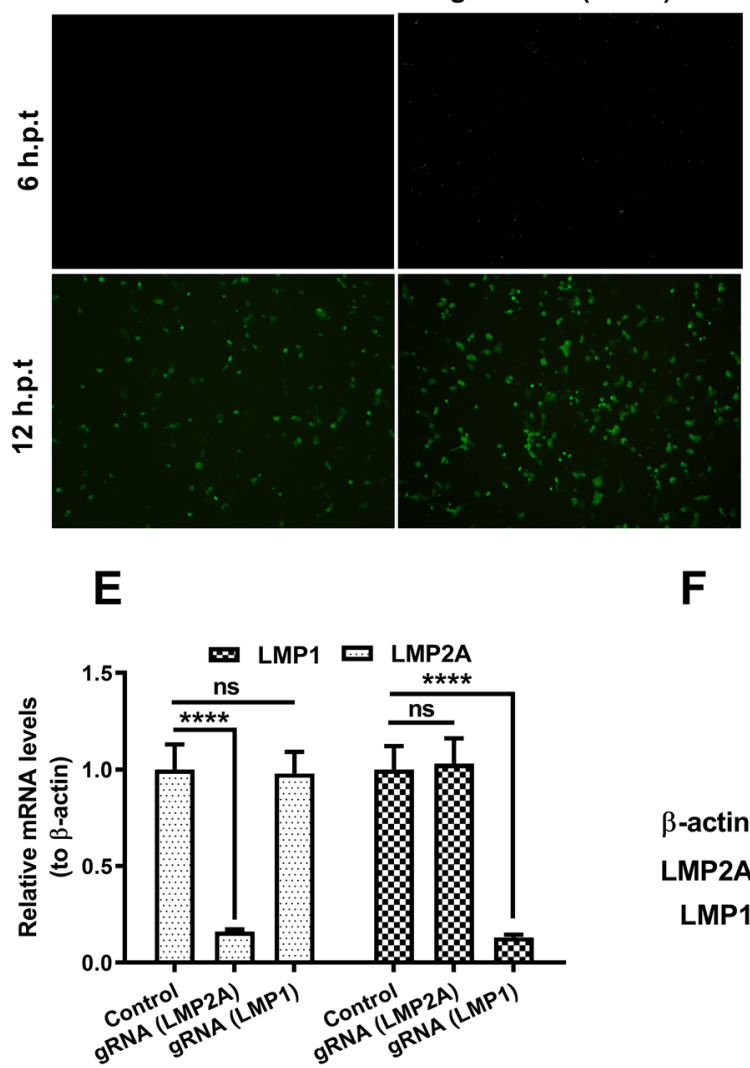

C o
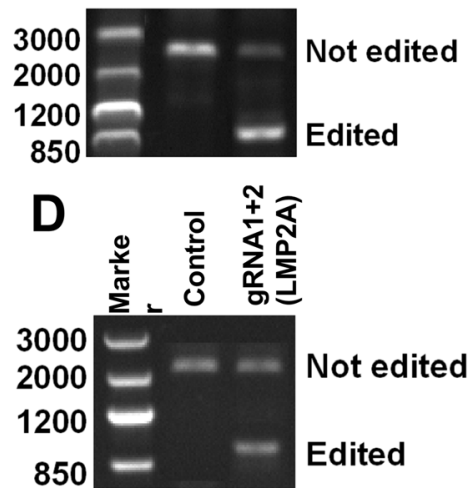

gRNA1+2 (LMP2A)

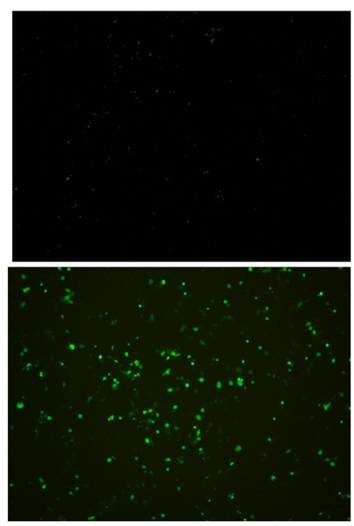

$\mathbf{F}$

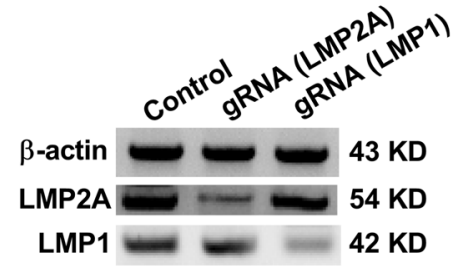

Fig. 3 Targeted CRISPR/Cas9 inhibition of EBV LMP1 and LMP2A genes in CNE-2 cells. a: Schematic depicting the target sites CRISPR/Cas9 editing in LMP2A (upper) and latent membrane protein 1 (LMP1) (lower) genes of the EBV genome. The target sites of gRNAs and binding sites of specific primers (LMP1-F, LMP1-R, LMP2A-F, and LMP2A-R) are indicated with arrows. b: Green fluorescence intensity in CNE-2 cells transfected with the LMP1-CRISPR/Cas9 or LMP2A-CRISPR/Cas9 plasmid for 6 and $24 \mathrm{~h}$. c and $\mathbf{d}$ : Editing effect of CRISPR/Cas9. CNE-2 cells were transfected with gRNA-Cas9 co-expression plasmids for $48 \mathrm{~h}$, and the total genomic DNA was extracted for PCR analysis withLMP1-(up) and LMP2A-specific (down) primers. e and $\mathbf{f}$ : Real-time quantitative PCR analysis (e) and western blotting (f) of LMP1 and LMP2A in 'LMP1 up' CNE-2 cells that were transfected with LMP1- or LMP2A-specific gRNA-Cas9 co-expression plasmids for 48 (for mRNA quantification) or 72 (for western blotting) hours. Experiments were independently performed in triplicate. ${ }^{*} p<0.05$; ${ }^{* * *} p<0.001$; ${ }^{* * * *} p<$ $0.0001 ; \mathrm{ns}$, no significance 
A

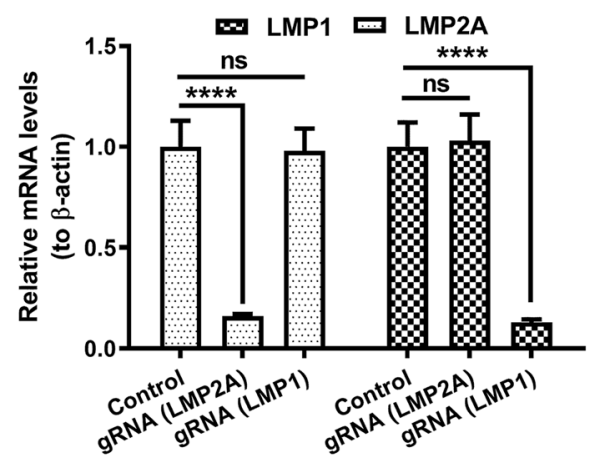

C

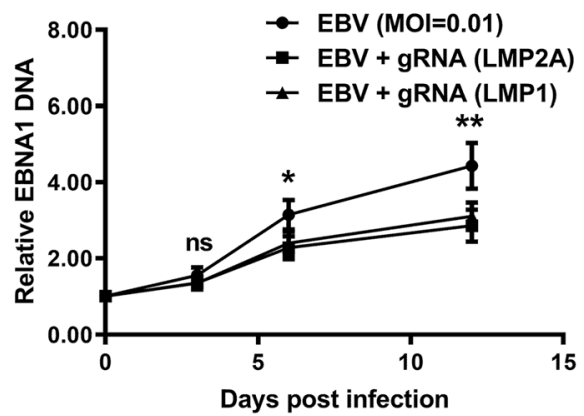

B

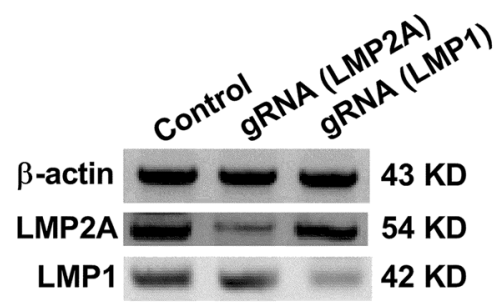

D

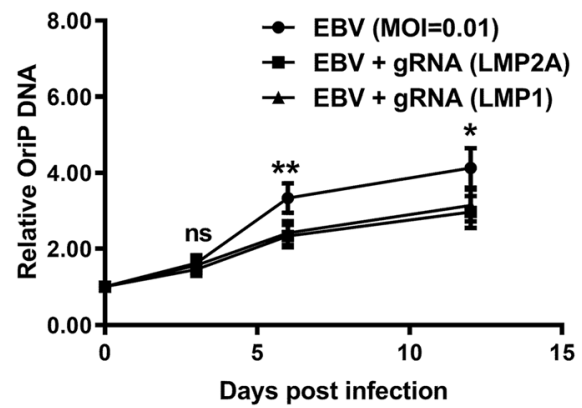

Fig. 4 Regulation by the CRISPR/Cas9-mediated knockout of LMP1 or LMP2A on the proliferation of EBV in CNE-2 cells. a: Real-time quantitative PCR analysis (a) and western blotting (b) of LMP1 and LMP2A in the 'LMP1 up' CNE-2 cells, which were transfected with LMP1- or LMP2A-specific gRNA-Cas9 co-expression plasmids for 48 (for mRNA quantification) or 72 (for western blotting) hours; $\mathbf{c}$ and $\mathbf{d}$ : Relative EBV growth in the 'LMP1 up' CNE-2 cells, post the CRISPR/Cas9-mediated knockout of LMP1 or LMP2A, with a multiplicity of infection (MOI) of 0.01 for 0, 3, 6, or 12 days; virus growth was determined via the quantification of viral DNA levels of EBNA1 (c) and OriP (d). Each value was an average for triple independent results. Statistical significance was showed as ${ }^{*} p<0.05,{ }^{* *} p<0.01,{ }^{* * *} p<0.0001$, ns: no significance

Both mRNA $(p<0.0001$ respectively, Fig. 4a) and protein (Fig. 4b) levels of LMP1 and LMP2A were markedly knocked down in CNE-2 cells at $24 \mathrm{~h}$ after EBV infection. In addition, the regulation by LMP1 or LMP2A editing on virus replication was also examined. The virus growth was evaluated with the viral DNA levels of EBNA1 and OriP. A significant reduction of EBNA1 (Fig. 4c) and of OriP (Fig. 4d) DNA $(p<0.05$ or $p<0.01$ for 6 or 12 -days postinfection) was caused by the knockout of either LMP. These results confirmed that the LMP1 or LMP2A knockout inhibited the EBV replication.

\section{CRISPR/Cas9mediated knockout of LMP1 inhibits CNE2 cell growth and elF4E activation}

CRISPR/Cas9-mediated knockout of both LMP1 and LMP2A was performed before measuring CNE-2 cell growth with colony forming assays. LMP1 knockout markedly decreased colony size in LMP1 up cells $(p<0.001$, the right column, Fig. 5 a, b). However,
LMP2A knockout did not have any effect (the middle column, Fig. 5 a, b). Significant colony size reduction was also observed in LMP1\&LMP2A up cells after LMP1 knockout $(p<0.01)$ rather than LMP2A knockout. Furthermore, knockout of either LMP did not influence colony size in LMP2A up cells. Therefore, CRISPR/Cas9-mediated knockout of LMP1 inhibited CNE-2 cell growth. In addition, we also examined the regulation by the CRISPR/Cas9-mediated knockout of LMP1 or of LMP2A on the eIF4E activation in CNE-2 cells. As indicated in Fig. 6a, the eIF4E mRNA was upregulated by the EBV infection from 3 to 12 days post-infection $(p<0.001)$. However, the eIF4E mRNA upregulation was blocked by either LMP1 knockout $(p<0.05$ or $\mathrm{p}<0.01)$ or by LMP2A knockout ( $<<0.05$, Fig. $6 \mathrm{~b})$. Such regulation was also confirmed at protein level. Not only LMP1 and LMP2A but also eIF4E were downregulated by the knockout of either LMP1 or LMP2A during EBV infection (Fig. 6c). 

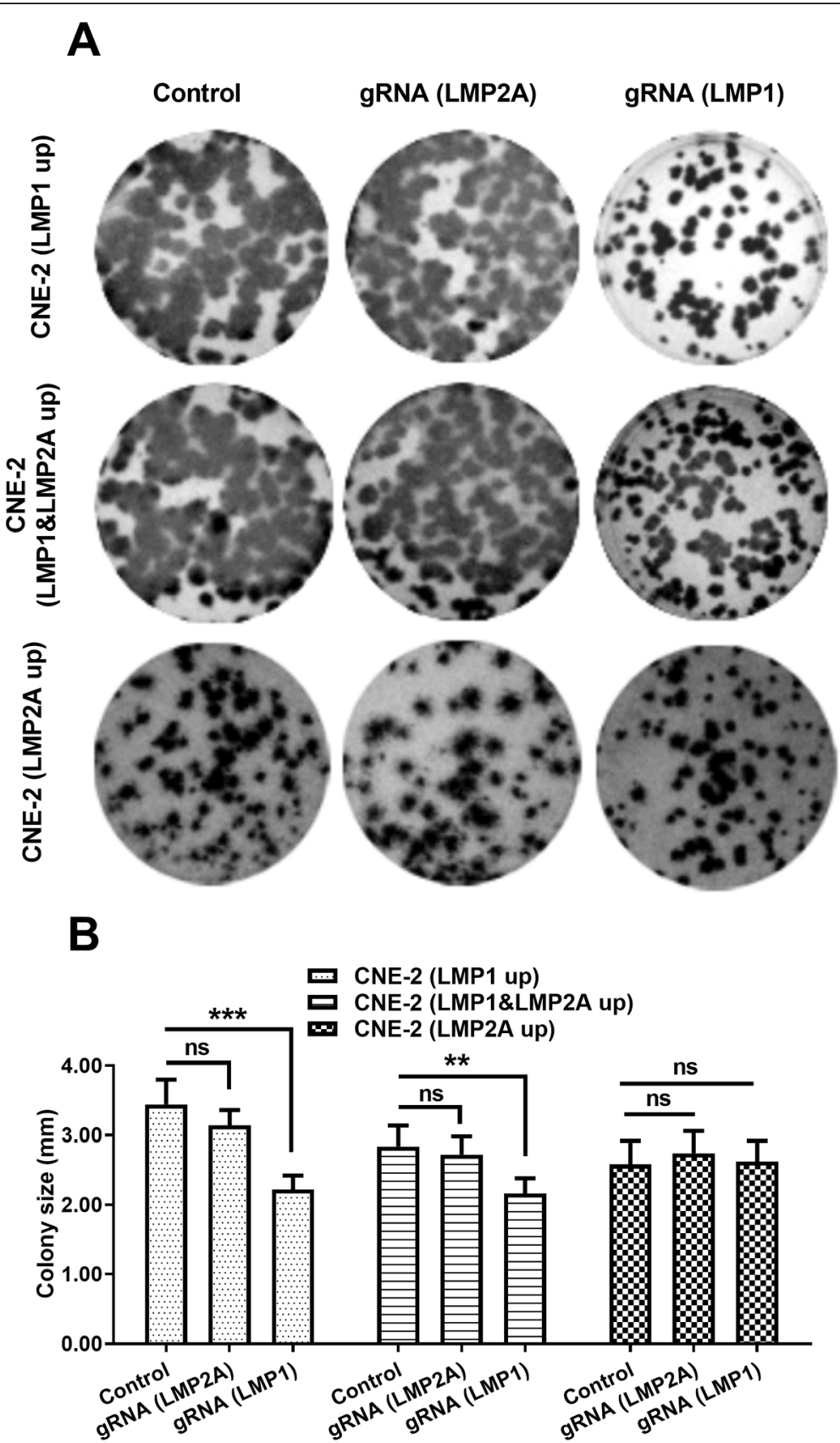

Fig. 5 CRISPR/Cas9-mediated knockout of LMP1 or LMP2A regulates CNE-2 cell growth. a and $\mathbf{b}$ : Colony formation (a) and size (b) of CNE-2 cells following the targeted knockout of LMP1 or LMP2A, via transfection with the LMP1- or LMP2A-specific gRNA-Cas9 co-expression plasmid. Each experiment was independently replicated three times. ${ }^{* *} p<0.01$; ${ }^{* *} p<0.001$; ns, no significance

\section{Discussion}

LMP1 upregulation was demonstrated to confer a proliferative advantage and the ability to resist apoptosis to NPC cells, via the interaction of LMP1 with cell cyclerelated molecules such as NF- $\mathrm{kB}$, JNK, and phosphatidylinositide 3-kinases (PI3K) [33]. In the present study, we confirmed in vitro that LMP1 promoted the proliferation of NPC CNE-2 cells at least partly via eIF4E upregulation. Previous studies indicated that LMP1 stimulated
eIF4E transcription [17] in LMP1-harboring NPC B95-8 cells. However, other signaling pathways, including HMGB1 [34], DNAzyme [18] and LKB1-AMPK [19] might also be involved. EBV infection was shown to cause a statistically significant overexpression of HMGB1 in NPC tissues, in association, in association with the malignant status of NPC. The HMGB1 upregulation was demonstrated to induce NPC cells proliferation, RAGE-dependently [34]. DNAzyme (DZ) 1 [18] 


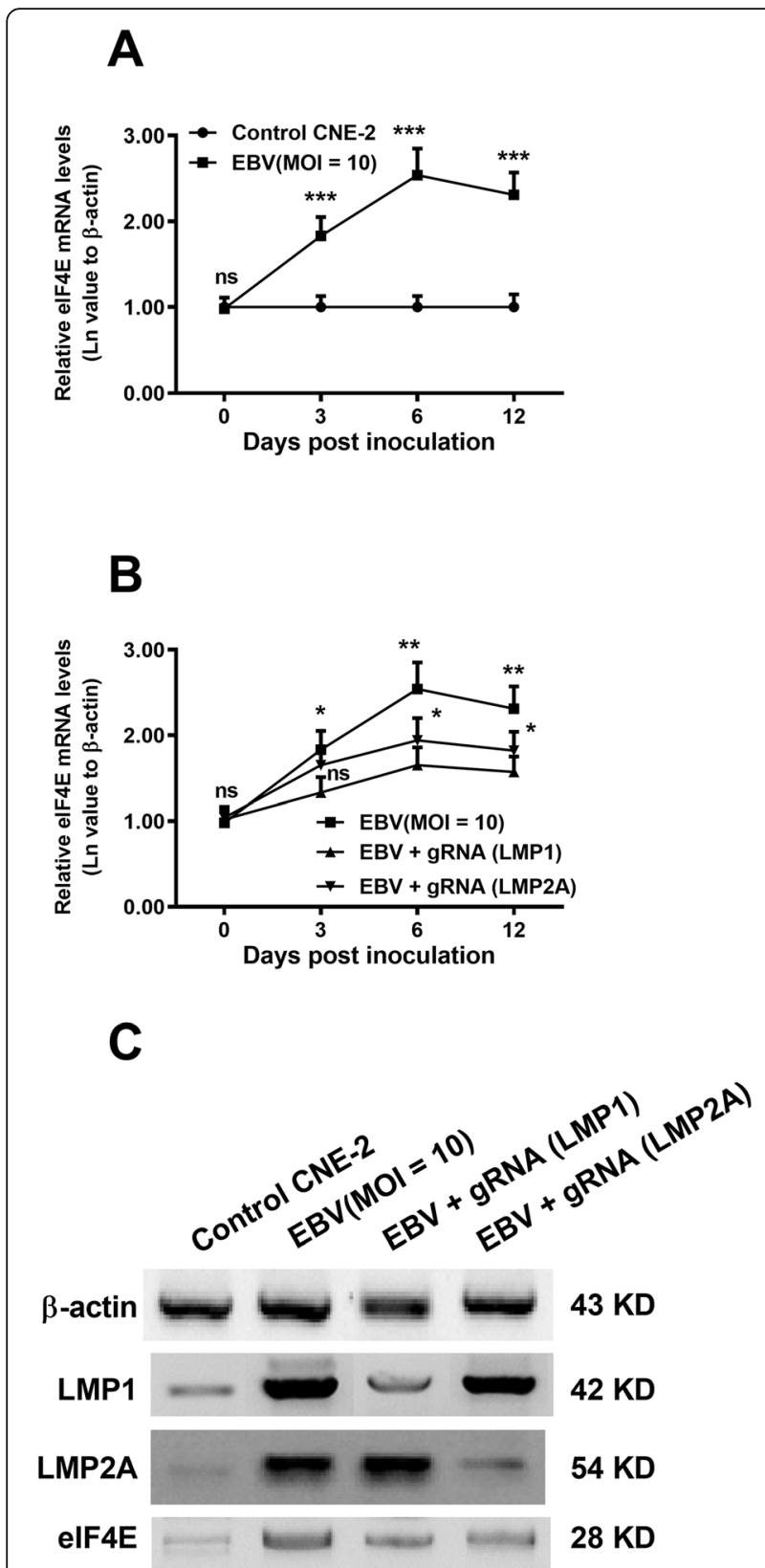

Fig. 6 CRISPR/Cas9-mediated knockout of LMP1 or LMP2A downregulates the elF4E activation in the EBV-infected CNE-2 cells. CRISPR/Cas9-mediated knockout of LMP1 or of LMP2A was performed in the CNE-2 cells, post the infection with $10 \mathrm{MOI} E B V$, then the mRNA and the protein levels of LMP1, LMP2A, and elF4E were examined by the method of Real-time quantitative PCR analysis (a) or of western blotting (b). Experiments were independently repeated in triplicate. ${ }^{* *} p<0.01$; ${ }^{* *} p<0.001$; ns, no significance

and LKB1-AMPK pathway [19] have also been found involved in such process via regulating cell cycle [18] or via regulating the proliferation and transformation of epithelial cells [19].
Advanced NPC has a high mortality rate [35]. Due to the anatomical complexity of the nasopharynx, surgical resection for NPC is technically challenging. Despite sensitivity to radiotherapy at early stages and encouraging clinical outcomes, it is still a challenge to administer for the same reason [36]. The roles of induction and adjuvant chemotherapy remain to be well defined [37, 38]. Given the overwhelming evidence of a robust oncogenic effect of EBV infection, targeting EBV infection might be a potential therapy. The present study and previous reports $[39,40]$ confirm the effective inhibition that anti-EBV infection strategies have on NPC cell growth. Notably, the coding sequence of the two latent viral proteins, LMP1 and LMP2A, integrates into the host genome and pose the ontogenetic effect for a lifelong time. It is a vital strategy to eliminate the two oncogenic genes from contaminated cells.

The CRISPR/Cas9 system derives from a prokaryotic antiviral immune system and can also effectively eliminate HPV genomic episomes from latent cells [28, 29]. Prevention of EBV infection by CRISPR/Cas9 mutagenesis was demonstrated in EBV-transformed Blymphoblastoid cells [41, 42], and this was directly related to LMP1 [43]. We constructed here LMP1- and LMP2A-targeted CRISPR/Cas9 systems to knockout the expression of either viral protein in CNE-2 cells, and we confirmed the anti-cancer effect of CRISPR/Cas9-mediated LMP1 knockout in CNE-2 cells. Such anti-cancer effect was associated with the blockage of eIF4E activation,

Our promising results indicate that CRISPR/Cas9-mediated knockout of either LMP1 or LMP2A can prevent EBV infection of CNE-2 cells. LMP1-mediated promotion to NPC cell growth can be effectively blocked by CRISPR/Cas9-mediated LMP1 knockout. Precise LMP1 knockout might be a promising method for targeted inhibition of EBV infection and NPC cell growth.

\section{Abbreviations}

CAT: Chloramphenicol acetyl transferase; c-JNK: c-JunN-terminal kinase: CRISPR: Clustered Regularly Interspaced Short Palindromic Repeats; EBERs: EBV-encoded small RNAs; EBNA1: Epstein-Barr nuclear antigen 1; EBV: Epstein-Barr virus; elF4E: Eukaryotic translation initiation factor 4E; EMT: Epithelial-mesenchymal transition; FBS: Fetal bovine serum; HIV: Human immunodeficiency virus; HMGB1: High mobility group box 1; HPV: Human papillomavirus; LKB1: Liver kinase B1; LMP1: Latent membrane protein 1; NFKB: Nuclear factor kappa-light-chain-enhancer of activated B cells; NPC: Nasopharyngeal carcinoma; RT-qPCR: Real-time quantitative polymerase chain reaction; sgRNA: single-guide RNA; STAT: Signal Transducer and Activator of Transcription

\section{Acknowledgements}

Dr.Lujun Li and Dejun Luo of Chongqing medical university provided purely technical help or writing assistance and general support.

\section{Authors' contributions}

$\mathrm{HH}$ carried out the molecular genetic studies, participated in the sequence alignment and drafted the manuscript. $\mathrm{HH}$ carried out the immunoassays. $\mathrm{HH}$ participated in the sequence alignment. $\mathrm{GH}$ participated in the design of 
the study and performed the statistical analysis,conceived of the study, and participated in its design and coordination and helped to draft the manuscript. All authors read and approved the final manuscript. All contributors who do not meet the criteria for authorship should be listed in an acknowledgements section.

\section{Funding}

The present study was supported by the grant from the Nature Fund of the First Affiliated Hospital of Chongqing Medical University (2018pyjj-01).

\section{Availability of data and materials}

All data in this manuscript were available upon a request.

\section{Ethics approval and consent to participate}

Participate were approved by Chongqing Medical University thics committee,reference number (CQMU201604752).

\section{Consent for publication}

All authors consent for publication.

\section{Competing interests}

The authors declare that they have no conflict of interests.

Received: 13 May 2019 Accepted: 20 September 2019 Published online: 30 October 2019

\section{References}

1. Wu L, Li C, Pan L. Nasopharyngeal carcinoma: a review of current updates. Exp Ther Med. 2018;15(4):3687-92.

2. Chua M, Wee J, Hui EP, Chan A. Nasopharyngeal carcinoma. Lancet. 2016; 387(10022):1012-24

3. Tsao SW, Tsang CM, To KF, Lo KW. The role of Epstein-Barr virus in epithelial malignancies. J Pathol. 2015;235(2):323-33.

4. Liebowitz D. Nasopharyngeal carcinoma: the Epstein-Barr virus association. Semin Oncol. 1994;21(3):376-81.

5. Kaye KM, Izumi KM, Mosialos G, Kieff E. The Epstein-Barr virus LMP1 cytoplasmic carboxy terminus is essential for B-lymphocyte transformation; fibroblast cocultivation complements a critical function within the terminal 155 residues. J Virol. 1995;69(2):675-83.

6. Huen DS, Henderson SA, Croom-Carter D, Rowe M. The Epstein-Barr virus latent membrane protein-1 (LMP1) mediates activation of NF-kappa B and cell surface phenotype via two effector regions in its carboxy-terminal cytoplasmic domain. Oncogene. 1995;10(3):549-60.

7. Zhang Y, Wang H, Liu Y, Wang C, Wang J, Long C, et al. Baicalein inhibits growth of Epstein-Barr virus-positive nasopharyngeal carcinoma by repressing the activity of EBNA1 Q-promoter. Biomed Pharmacother. 2018; 102:1003-14.

8. Wang L, Tian WD, Xu X, Nie B, Lu J, Liu X, et al. Epstein-Barr virus nuclear antigen 1 (EBNA1) protein induction of epithelial-mesenchymal transition in nasopharyngeal carcinoma cells. Cancer-Am Cancer Soc. 2014;120(3):363-72.

9. Turunen A, Rautava J, Grenman R, Syrjanen K, Syrjanen S. Epstein-Barr virus (EBV)-encoded small RNAs (EBERs) associated with poor prognosis of head and neck carcinomas. Oncotarget. 2017;8(16):27328-38.

10. Pfeffer S, Zavolan M, Grasser FA, Chien M, Russo JJ, Ju J, et al. Identification of virus-encoded microRNAs. Science. 2004;304(5671):734-6.

11. Chang Y, Cheng SD, Tsai CH. Chromosomal integration of Epstein-Barr virus genomes in nasopharyngeal carcinoma cells. Head Neck. 2002;24(2):143-50.

12. Kaschka-Dierich C, Adams A, Lindahl T, Bornkamm GW, Bjursell G, Klein G, et al. Intracellular forms of Epstein-Barr virus DNA in human tumour cells in vivo. Nature. 1976;260(5549):302-6.

13. Eliopoulos AG, Gallagher NJ, Blake SM, Dawson CW, Young LS. Activation of the p38 mitogen-activated protein kinase pathway by Epstein-Barr virusencoded latent membrane protein 1 coregulates interleukin- 6 and interleukin-8 production. J Biol Chem. 1999;274(23):16085-96.

14. Eliopoulos AG, Young LS. Activation of the cJun N-terminal kinase (JNK) pathway by the Epstein-Barr virus-encoded latent membrane protein 1 (LMP1). Oncogene. 1998;16(13):1731-42.

15. Bentz GL, Whitehurst $C B$, Pagano JS. Epstein-Barr virus latent membrane protein 1 (LMP1) C-terminal-activating region 3 contributes to LMP1mediated cellular migration via its interaction with Ubc9. J Virol. 2011;85(19): 10144-53.
16. He J, Tang F, Liu L, Chen L, Li J, Ou D, et al. Positive regulation of TAZ expression by EBV-LMP1 contributes to cell proliferation and epithelialmesenchymal transition in nasopharyngeal carcinoma. Oncotarget. 2017; 8(32):52333-44.

17. Zhao Y, Pang TY, Wang Y, Wang S, Kang HX, et al. LMP1 stimulates the transcription of elF4E to promote the proliferation, migration and invasion of human nasopharyngeal carcinoma. FEBS J. 2014;281(13):3004-18.

18. Ma X, Xu Z, Yang L, Xiao L, Tang M, Lu J, et al. EBV-LMP1-targeted DNAzyme induces DNA damage and causes cell cycle arrest in LMP1positive nasopharyngeal carcinoma cells. Int J Oncol. 2013;43(5):1541-8.

19. Lo AK, Lo KW, Ko CW, Young LS, Dawson CW. Inhibition of the LKB1-AMPK pathway by the Epstein-Barr virus-encoded LMP1 promotes proliferation and transformation of human nasopharyngeal epithelial cells. J Pathol. 2013, 230(3):336.

20. Cong L, Ran FA, Cox D, Lin S, Barretto R, Habib N, et al. Multiplex genome engineering using CRISPR/Cas systems. Science. 2013;339(6121):819-23.

21. Jinek M, Chylinski K, Fonfara I, Hauer M, Doudna JA, Charpentier E. A programmable dual-RNA-guided DNA endonuclease in adaptive bacterial immunity. Science. 2012;337(6096):816-21.

22. Mali P, Yang L, Esvelt KM, Aach J, Guell M, DiCarlo JE, et al. RNA-guided human genome engineering via Cas9. Science. 2013;339(6121):823-6.

23. Doench JG, Hartenian E, Graham DB, Tothova Z, Hegde M, Smith I, et al Rational design of highly active sgRNAs for CRISPR-Cas9-mediated gene inactivation. Nat Biotechnol. 2014;32(12):1262-7.

24. Canver MC, Smith EC, Sher F, Pinello L, Sanjana NE, Shalem O, et al. BCL11A enhancer dissection by Cas9-mediated in situ saturating mutagenesis. Nature. 2015;527(7577):192-7.

25. Zhu S, Li W, Liu J, Chen CH, Liao Q, Xu P, et al. Genome-scale deletion screening of human long non-coding RNAs using a paired-guide RNA CRISPR-Cas9 library. Nat Biotechnol. 2016;34(12):1279-86.

26. Soppe JA, Lebbink RJ. Antiviral Goes viral: harnessing CRISPR/Cas9 to combat viruses in humans. Trends Microbiol. 2017:25(10):833-50.

27. White MK, Hu W, Khalili K. The CRISPR/Cas9 genome editing methodology as a weapon against human viruses. Discov Med. 2015;19(105):255-62.

28. Lao YH, Li M, Gao MA, Shao D, Chi CW, Huang D, et al. HPV Oncogene manipulation using nonvirally delivered CRISPR/Cas9 or Natronobacterium gregoryi Argonaute. Adv Sci (Weinh). 2018;5(7):1700540.

29. Ehrke-Schulz E, Schiwon M, Leitner T, David S, Bergmann T, Liu J, et al. CRISPR/Cas9 delivery with one single adenoviral vector devoid of all viral genes. Sci Rep. 2017;7(1):17113.

30. Kaminski R, Chen Y, Fischer T, Tedaldi E, Napoli A, Zhang Y, et al. Elimination of HIV-1 genomes from human T-lymphoid cells by CRISPR/Cas9 gene editing. Sci Rep. 2016;6:22555.

31. van Diemen FR, Kruse EM, Hooykaas MJ, Bruggeling CE, Schurch AC, van Ham PM, et al. CRISPR/Cas9-mediated genome editing of herpesviruses limits productive and latent infections. PLoS Pathog. 2016;12(6):e1005701.

32. Wang J, Quake SR. RNA-guided endonuclease provides a therapeutic strategy to cure latent herpesviridae infection. Proc Natl Acad Sci U S A. 2014;111(36):13157-62

33. Xiao L, Hu ZY, Dong X, Tan Z, Li W, Tang M, et al. Targeting Epstein-Bar virus oncoprotein LMP1-mediated glycolysis sensitizes nasopharyngeal carcinoma to radiation therapy. Oncogene. 2014;33(37):4568-78.

34. Zhu X, Sun L, Wang Y. High mobility group box 1 (HMGB1) is upregulated by the Epstein-Barr virus infection and promotes the proliferation of human nasopharyngeal carcinoma cells. Acta Otolaryngol. 2016;136(1):87-94.

35. Faivre S, Janot F, Armand JP. Optimal management of nasopharyngeal carcinoma. Curr Opin Oncol. 2004;16(3):231-5.

36. Zackrisson B, Mercke C, Strander H, Wennerberg J, Cavallin-Stahl E. A systematic overview of radiation therapy effects in head and neck cancer. Acta Oncol. 2003:42(5-6):443-61.

37. Yoshizaki T, Kondo S, Murono S, Endo K, Tsuji A, Nakanishi Y, et al. Progress and controversy for the role of chemotherapy in nasopharyngeal carcinoma. Jpn J Clin Oncol. 2015;45(3):244-7.

38. Tan WL, Tan EH, Lim DW, Ng QS, Tan DS, Jain A, et al. Advances in systemic treatment for nasopharyngeal carcinoma. Chin Clin Oncol. 2016;5(2):21.

39. Wu Q, Han T, Sheng X, Zhang N, Wang P. Downregulation of EB virus miRBART4 inhibits proliferation and aggressiveness while promoting radiosensitivity of nasopharyngeal carcinoma. Biomed Pharmacother. 2018; 108:741-51

40. Yiu S, Hui KF, Choi CK, Kao R, Ma CW, Yang D, et al. Intracellular Iron chelation by a novel compound, C7, reactivates Epstein(-)Barr virus (EBV) 
lytic cycle via the ERK-autophagy Axis in EBV-positive epithelial cancers. Cancers (Basel). 2018;10(12):505.

41. Greenfeld H, Takasaki K, Walsh MJ, Ersing I, Bernhardt K, Ma Y, et al. TRAF1 coordinates Polyubiquitin signaling to enhance Epstein-Barr virus LMP1mediated growth and survival pathway activation. PLoS Pathog. 2015;11(5): e1004890.

42. Ohashi M, Holthaus AM, Calderwood MA, Lai CY, Krastins B, Sarracino D, et al. The EBNA3 family of Epstein-Barr virus nuclear proteins associates with the USP46/USP12 deubiquitination complexes to regulate lymphoblastoid cell line growth. PLoS Pathog. 2015;11(4):e1004822.

43. Yuen KS, Wang ZM, Wong NM, Zhang ZQ, Cheng TF, Lui WY, Chan CP, Jin DY, et al. Suppression of Epstein-Barr virus DNA load in latently infected nasopharyngeal carcinoma cells by CRISPR/Cas9. Virus Res. 2018;244:296-303.

\section{Publisher's Note}

Springer Nature remains neutral with regard to jurisdictional claims in published maps and institutional affiliations.

Ready to submit your research? Choose BMC and benefit from:

- fast, convenient online submission

- thorough peer review by experienced researchers in your field

- rapid publication on acceptance

- support for research data, including large and complex data types

- gold Open Access which fosters wider collaboration and increased citations

- maximum visibility for your research: over $100 \mathrm{M}$ website views per year

At $\mathrm{BMC}$, research is always in progress.

Learn more biomedcentral.com/submissions 